\title{
Launching Vehicle Acoustic Data Compression Study Using Lossy Audio Formats
}

\author{
Guilherme Coelho da Silva Stanisce Corrêa ${ }^{1 *}$, Rogério Pirk ${ }^{1,2}$, Marcelo da Silva Pinho ${ }^{1}$
}

\author{
Correa GCSS iD https: / /orcid.org/0000-0002-6379-8086 \\ Pirk R (D) https://orcid.org/0000-0002-9963-1333 \\ Pinho MS (D) https: / / orcid.org/0000-0002-9393-8473
}

\section{How to cite}

Correa GCSS; Pirk R; Pinho MS (2020) Launching Vehicle Acoustic Data Compression Study Using Lossy Audio Formats. J Aerosp Technol Manag, 12: e2920. https://doi. org/10.5028/jatm.v12.1124

ABSTRACT: The field of data compression has evolved over the last decades. In this way, several techniques to reduce the amount of acquired data from the sensor required to be transmitted have been developed. Those techniques are usually classified by lossless or lossy, where, for the lossless techniques, all acquired data is recovered, while the lossy techniques introduce errors to these data. Each of these techniques presents advantages and drawbacks, being the analyst responsible for choosing the appropriate technique for a specific application. This work presents a comparative study using lossy audio formats to be applied on a launch vehicle on-board acoustic data. The Opus format achieved a higher compression rate in comparison with standard compression techniques by saving up to 254 times the required amount of data to be transmitted through a telemetry link on launcher vehicle, and the lowest discrepancy from original data measured by the mean square error metric.

KEYWORDS: Data compression; Lossy; Aerospace; Launcher; Telemetry.

\section{INTRODUCTION}

The monitoring of physical parameters of spacecraft during the launch, which can prevent equipment failures that could lead to human loss or payload damage, is a critical task. One of the most impressive fatal accident in the space field happened during the launch of the space shuttle Challenger, where it leads to the death of the entire crew after 73 seconds after lift-off (NASA 1986). In this catastrophic accident, an investigation commission has been created, and the many physical parameters measured during these 73 seconds of flight were extensively analyzed.

The lengthy analysis of the measured data has concluded that the accident caused by a structural failure in the hydrogen tank and actions to mitigate the cause of the accident for the next Challenger flights were proposed. On the other side, during the development of the space launcher Falcon 9, the American company SpaceX has performed 16 test flights in the period between 2013 and 2016 , aiming the development of a reusable rocket. After several accident occurrences in the flight tests aiming at the challenging vehicle recovery, the protoflight number 23 has successfully achieved the primary goal of the reusability of the referred spacecraft (Clarke 2016). The risks involved in rocketry are well known, and these achievements would not be fulfilled without the on-board measurements of hundreds of physical parameters, usually done by tailored telemetry systems. Such famous cases of the spatial industry can illustrate how vital are the on-board telemetry systems as a rocket subsystem and how they play a crucial role during spacecraft launches.

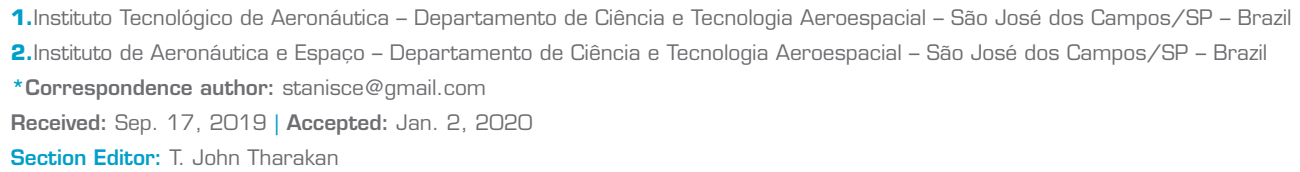


The on-board vibration and mechanical shock measurements allocate large capacity amounts of telemetry systems. Such a simple allocated memory assessment usually takes launcher vehicle managers to eliminate these expensive (in terms of allocated memory) highfrequency measurements, since an overview of the low-frequency measurements along the different compartments of the launcher as temperatures, motors and ignitors static pressures, guidance and control systems are paramount to gather enough information to assess real-time flight trajectory, launcher performances, compartments heating and possible accident causes. On the other side, technical decisions eliminating high-frequency measurements affect sub-systems dynamical analysis as well as on-board equipment functionality analyses, in case of failure. Also, these sizeable spectral content data, characterized by vibration, mechanical shock and acoustic measurements are essential to assess structural dynamic excitations and responses as well as to characterize acoustic environments.

Regarding the acoustics and vibroacoustics of launchers, the external and internal dynamic environments generated during a flight mission must be known. Internal vibrations of fairings and equipment bays, induced by external acoustic excitations at different moments of the flight as lift-off, transonic flight and maximum dynamic pressure flight are essential data to specify qualification and acceptance tests of on-board equipment and payloads (Arenas and Margasahayam 2006; Pirk and Souto 2015). The dynamic operational environments during the flight are monitored aiming at validating virtual prototypes, built by using deterministic and statistical vibroacoustic prediction techniques, such as the statistical energy analysis (SEA).

The need for using a compressor for the on-board high-frequency data measurements seems to be appropriate as described above. Nevertheless, the reliability of the recovered signal and allocated memory are essential parameters to be accounted for in this task. Besides, as a matter of keeping the generalities of the analyzed signal, measured in-flight data should be compressed. However, real flight acoustic signals are not available due to confidentiality issues. In this framework, the sound pressure levels (SPL) generated during the S40 propeller burning test campaign is analyzed by using the data compression techniques, without loss of generalities (mainly signal magnitude and sampling frequency).

\section{DATA COMPRESSION ON AEROSPACE APPLICATION}

This section describes three cases where data compression is used in the aerospace area for sound and vibration signals, outlining the techniques applied and its results.

\section{ADAPTIVE COMPRESSION SCHEMES FOR HOUSEKEEPING DATA}

A group of researchers from the German space agency have developed a data compression scheme for housekeeping data on launchers and satellites (Meß et al. 2017).

To perform the data compression, they have used the discrete cosine transform (DCT), and discrete wavelet transform (DWT) to remove redundancies in the original data. Then, this information is quantized to limit the number of bits allocated to store each coefficient. Finally, the data is encoded and sent to the ground station, where once the data is received, decoding is applied; subsequently, an inverse transformation is computed, and the sensor data is reconstructed.

The results using this data compression scheme were by the factor of $96.5 \%$ for rapidly oscillating vibration sensors, and $99.5 \%$ for the smooth output of temperature sensors.

\section{A LOSSLESS COMPRESSION ALGORITHM FOR VIBRATION DATA OF SPACE SYSTEMS}

This study from Indian researchers based on a lossless compression scheme where the vibration signal is split into two sets, then it applies a custom algorithm. Finally, run-length encoding is done (Abraham et al. 2016).

According to their study, the proposed data compression scheme can reach compression rate up to 4.15 for a signal without noise, and 2.75 for a noise signal, by relying on the vibration signal has several defined patterns.

Since the study has the assumption of patterns, their system uses a dictionary with the position where a given pattern has occurred. By using the location instead of the signal pattern, they saved an amount of data, which is sent to the run-length encoding block to perform the lossless compression. 


\section{AN EFFICIENT COMPRESSION ALGORITHM AND A NOVEL FILE FORMAT FOR SATELLITE VIBRATION TEST DATA}

The group of researchers from the Indian Space Research Organisation Satellite Center had to solve the problem of storing vibration data acquired on test benches. During the tests, up to $55 \mathrm{~GB}$ data were acquired through the data acquisition system and stored for postprocessing (Nagendra et al. 2013).

The solution was the creation of a compressed file format, using wavelet transform, a frequency estimation algorithm and arithmetic coding. The results obtained in this case were a data size reduction up to $95 \%$ and a mean square error below $0.01 \%$ in comparison to the original data.

\section{AUDIO COMPRESSION}

The area of audio compression has been studied over the past decades mainly focusing on how to improve the entropy coding without affecting human perception (Bosi and Goldberg 2003; Fastl and Zwicker 2007; McLoughlin 2016; Hill 2018).

From the many different lossy audio formats available in the market, the main developing groups (MPEG - Moving Picture Experts Group and Xiph.Org Foundation) and its formats are discussed in this section.

\section{MOVING PICTURE EXPERTS GROUP (MPEG)}

The International Standards Organization (ISO) and the International Electrotechnical Commission (IEC) organizations established in 1988 a committee to study and defined coding for audio and video called ISO/IEC/MPEG.

The MPEG was the first to develop a complete system-level standard solution for digital audio and video. The MPEG-1 standard includes a part for video compression, another for audio compression and a third for the audiovisual stream (Watkinson 2004). The MPEG-1 audio standard (ISO 1993a) describes a perceptual audio coding algorithm designed for general audio signals. This standard had application in areas such as digital compact cassette (DCC), digital audio and video broadcasting (DAB and DVB), and portable devices, among others. As a matter of explanation on the subject, MPEG-1 and MPEG-2 are defined as audio and video coding systems, whereas the layers I (MP1), II (MP2), and III (MP3) of MPEG are associated to audio coding, thus being the body of this study. The MP2, MP3, and advanced audio coding (AAC) audio formats defined by the MPEG group were selected because they are based on perceptual coding format, which means that part of the audio listened by the human ear is discarded following the psychoacoustic model. In practice, the frequency range best perceived by the human ear remains the same up to $4 \mathrm{kHz}$, and outside this range a quantization factor is applied to reduce the quality of the signal, thus requiring fewer data to be stored.

\section{MPEG-1 Layer II (MP2)}

The MPEG-1 Layer II or MP2 format defined by the ISO/IEC 11172-3:1993 (ISO 1993b) and ISO/IEC 13818-3:1998 (ISO 1998) standards as a lossy audio compression format, which had been created according to the MUSICAM audio codec (Dehery et al. 1991). The MUSICAM audio codec was designed to work in a wide range of applications, from broadcasting and telecommunication, up to digital storage media.

The MP2 is a sub-band audio encoder which uses a low-delay filter bank producing 32 frequency domain components. If any sub-band deemed to be imperceptible, then that sub-band is not transmitted.

\section{MPEG-1 Layer III (MP3)}

The MPEG-1 Layer III or MP3 format defined by ISO/IEC 11172-3:1993 (ISO 1993b), is considered to be the most complex layer of this ISO standard. This format is the evolution of the MP2 format with the addition of a hybrid transform, nonuniform quantization with entropy coding, and different window sizes. This has allowed increasing the compression rate from 8:1 to 12:1 (Madisetti 2010). 
An essential feature of MP3 is that this format is the only layer in the ISO/IEC 11172-3:1993 standard which supports variable bitrate.

\section{Advanced audio coding (AAC)}

The AAC audio format (ISO 2004) was created to be the evolution of the MPEG-1 standard with multichannel extension and supporting audio coding systems at lower frequencies (16 to $24 \mathrm{kHz}$ ). However, after a call for proposals done in 1993, it was defined that this standard would not have backward compatibility, thus being renamed to MPEG advanced audio coding, or simply AAC.

The AAC format had the primary objective to define a higher quality multichannel standard better than MPEG-1, delivering the highest possible performance using newly developed tools that could not be used in any backwards compatible standard.

\section{XIPH.ORG FOUNDATION}

The Xiph.Org Foundation (Xiph.Org Foundation 2016) is an open-source community based on multimedia related projects, such as Vorbis, Opus, Free Lossless Audio Codec (FLAC), Speex, among others. This foundation has been created as an alternative to commercial solutions designed by the MPEG group, where the MP3 audio format plays an important role. In September 1998, the Fraunhofer IIS Society sent a letter to small commercial and open source MPEG layer 3 (or as better known as MP3) developed projects requesting royalties for any commercial players, encoders, and work of art sold using that format. The response to the necessity of paying royalties to the multimedia related project was the creation of open-source alternatives like Vorbis from this community. Since its creation, other audio (FLAC, Opus, Speex), video (Daala, Theora), and streaming (Icecast) formats have been designed by this community. In this paper, the Vorbis and Opus audio formats are described in further details as they are the lossy formats supported by the Xiph.Org community. The FLAC audio format is lossless, and the Speex format focuses on speech compression.

\section{Vorbis}

The Vorbis (Xiph.Org Foundation 2015; Barbato 2008) audio compression format is an open-source lossy audio format created by Xiph.org organization to replace proprietary audio format MP3, due to its similar features, which has been studied over a decade (Selasky 2006).

The Vorbis is a fully open, nonproprietary, patent-and-royalty-free, general purpose compressed audio format for mid to high quality ( $8-48.0 \mathrm{kHz}, 16+$ bit, polyphonic) audio and music at fixed and variable bitrates from 16 to $128 \mathrm{kbps} / \mathrm{channel}$.

This format was designed to reduce sufficient bitrate by both eliminating interchannel redundancy and eliminating stereo image information labelled inaudible or undesirable according to spatial psychoacoustic model.

\section{Opus}

The Opus (Valin et al. 2012) is an audio format which is based on using different layers according to the desired application. For a speech, the linear prediction (LP) layer is used by encoding more efficiently the signal using the code-excited linear prediction (CELP) technique in comparison to transforming domain techniques. The modified discrete cosine transform (MDCT) is used by another layer to encode music and higher speech frequencies.

At any given time, either the LP layer, the MDCT layer, or both, maybe active, thus making this format the most flexible in comparison to the other lossy formats presented in this study. This format supports the bitrates from 6 to $510 \mathrm{kbps}$.

\section{METHODOLOGY}

The acoustic pressure data recorded during the rocket motor burning test was imported to MATLAB as a voltage signal within 0 to 5 Volts magnitude range. Using the microphone sensitivity $(\mathrm{mV} / \mathrm{Pa})$, the pressure captured by this sensor during the operation of the S-40 motor was converted into engineering unit (Pascal) and the signal time history curve has been built. As a consequence, the spectral content of this acoustic signal was determined by using a simple fast Fourier transform (FFT) operation. 


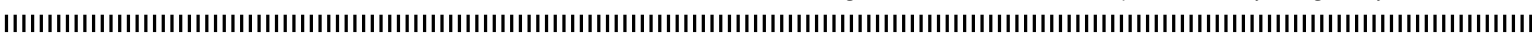

Since the pressure vs frequency curve had to be converted to audio format via audiowrite function to generate the lossless WAV file, the maximum pressure value was saved to a variable, and the curve was rescaled to be within unitary range. In this procedure, the data outside that unitary range was clipped by the audiowrite function.

In acoustic measurements, high sampling rates are needed, compared to low-frequency physical parameters (i.e. temperature and strength) or even to vibration measurements sampling rates. The adopted sampling frequency for the acoustic measurements was originally $32,768 \mathrm{~Hz}$ (about $4 \times$ the frequency of interest); however, due to constraints on exporting these data to a lossy format, the signal was resampled to $44,100 \mathrm{~Hz}$. The resampling was an important step to be able to generate audio in different lossy formats; otherwise, only Opus and Vorbis works with the original sampling rate. After resampling the acoustic data, a lossless WAV file was generated. This file was used as input to create the files for the different compression AAC, MP2, MP3, Opus, and Vorbis lossy formats for constant and variable bitrate configurations.

In order to avoid the use of different bitrates than expected during the generation of the referred lossy formats, the selected bitrate was set within 32 to $500 \mathrm{Kbps}$ range, using a constant variable setup. This is a critical setting procedure that makes the comparison among other invalid formats, which allowed to measure the performance for a given bitrate.

By adopting a constant bitrate configuration, the lossy format may not work as intended, which could lead to reducing even further the number of bits. In order to evaluate the performance of each lossy audio format, the files were recreated using variable bitrate.

After creating all the lossy files, the data was imported and stored in MATLAB variable structure, and once all structures were correctly placed, the calculations were done. It is essential to highlight that the voltage magnitude of the signal was scaled to pressure engineering unit (Pascal) by multiplying the maximum pressure value (in Volts) by the microphone sensitivity, given in the calibration chart of the sensor.

\section{METRICS}

This section provides background information in the metrics used to compare the performance of the different audio formats used in the study.

\section{MEAN SQUARE ERROR (MSE)}

The comparison between the original and compressed data was made using the mean square error (MSE) metric, which is a value that gives information regarding the discrepancy between two compared quantities.

The MSE is calculated as presented in Eq. 1.

$$
M S E=\frac{1}{N} \sum_{i=1}^{N}\left(x_{i}-\hat{x}_{i}\right)^{2}
$$

where $x i$ is the recorded raw data, $\hat{x} i$ is the compressed data, and $N$ is the number of samples.

\section{COMPRESSION RATE [CR]}

The compression rate (CR) is calculated by dividing the original file size by the compressed file size in bytes, as presented in Eq. 2 .

$$
C R=\frac{\text { OriginalFileSize }}{\text { CompressedFileSize }}
$$




\section{SOUND PRESSURE LEVEL [SPL]}

The primary information in vibroacoustic studies is retrieved from the measured acoustic pressure data, represented in terms of sound pressure levels (SPL) and their spectral distribution along with the frequency of interest (in structural acoustics up to $8,000 \mathrm{~Hz}$ ). Sound pressure level is a logarithm scale, which is defined as described in Eq. 3, where the ratio of the effective pressure $(P)$ generated by the sound, relative to a reference value $\left(P_{r e f} 20 \times 10^{-6}\right)$, usually given in Pascal $(\mathrm{Pa})$, is accounted and results are expressed in the $\mathrm{dB}$ scale:

$$
S P L=20 \log _{10}\left(\frac{P}{P_{r e f}}\right)
$$

The SPL values obtained by the one-third centre frequency bands were compared by calculating the standard deviation across the bitrate range on each audio format. By computing the standard deviation, it is possible to understand how the bitrate affects the SPL results for a given frequency band.

\section{RESULTS}

In this section, the MSE, CR, and SPL results are compared and analyzed using constant bitrate (CBR) and variable bitrate (VBR) configurations for the AAC, MP2, MP3, Opus, and Vorbis lossy audio formats.

\section{CONSTANT BITRATE (CBR) ANALYSIS}

The results presented in this section compares the MSE, CR, and SPL values for the same bitrate range for AAC, MP2, MP3, Opus, and Vorbis audio formats. The bitrate values in kbps obtained by CBR configuration were 64, 80, 96, 112, 128, 160, 192, 224 , and 256.

\section{Mean square error (MSE) comparison}

The Opus format has achieved the best results across the entire bitrate, reaching values lower than $1.44 \times 10^{-5}$ for the highest bitrate, and $19.46 \times 10^{-5}$ in the lowest bitrate. The Vorbis had the second-best results across the entire bitrate range, achieving $5.22 \times 10^{-5}$ and $51.02 \times 10^{-5}$ for the higher and lower bitrates, respectively.

On the other hand, the AAC, MP2, and MP3 obtained the worst results across the entire bit range. Figure 1 shows the mean square error for each lossy audio format.

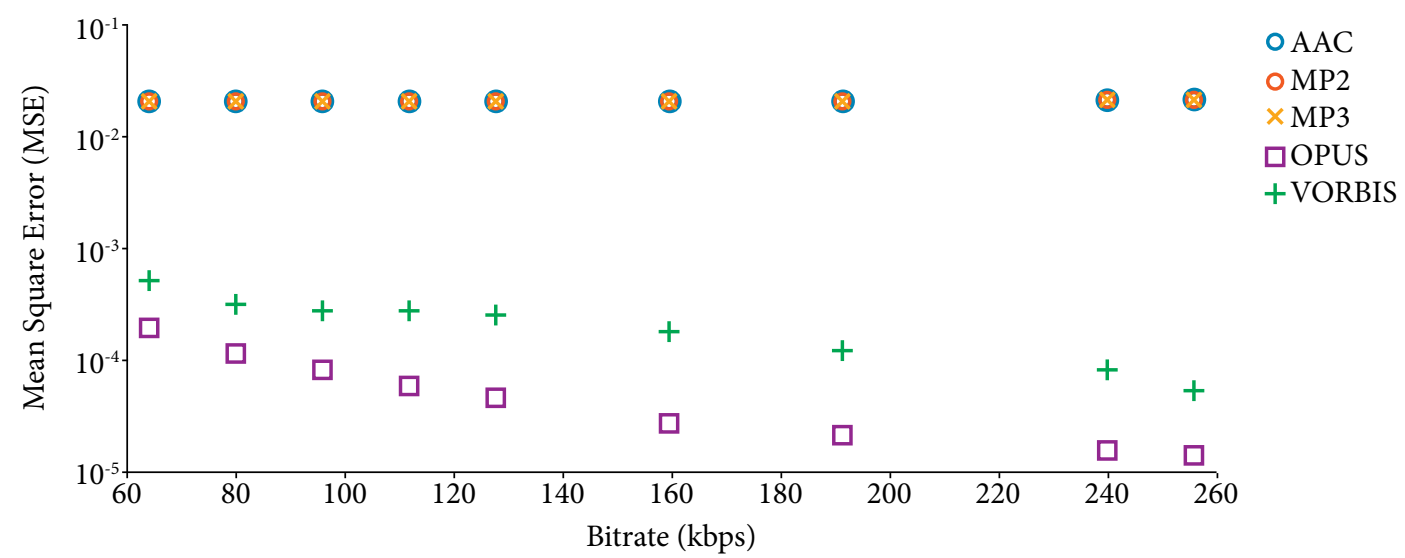

Figure 1. Mean square error for each audio format. 


\section{Compression rate (CR) comparison}

Figure 2 displays the compression rate for each audio format by bitrate. The Vorbis audio format has achieved the best compression across the entire bitrate range, reaching 33:1, saving up to $97 \%$ storage data. On the other hand, the AAC format presented the lowest compression rate for up to $132 \mathrm{kbps}$, however above this bitrate all the other formats achieved a similar result.

\section{Sound pressure level [SPL]}

The computed standard deviation from the SPL results shows that for frequencies below $800 \mathrm{~Hz}$, the Opus, MP2, and AAC presents the best results. Above $800 \mathrm{~Hz}$, only Opus has achieved the lowest results.

The MP3 results were the worst up to $2500 \mathrm{~Hz}$, where above this frequency Vorbis presented an increase in the standard deviation. The results above $8000 \mathrm{~Hz}$ are not considered in this analysis since it is outside the interest range of the study.

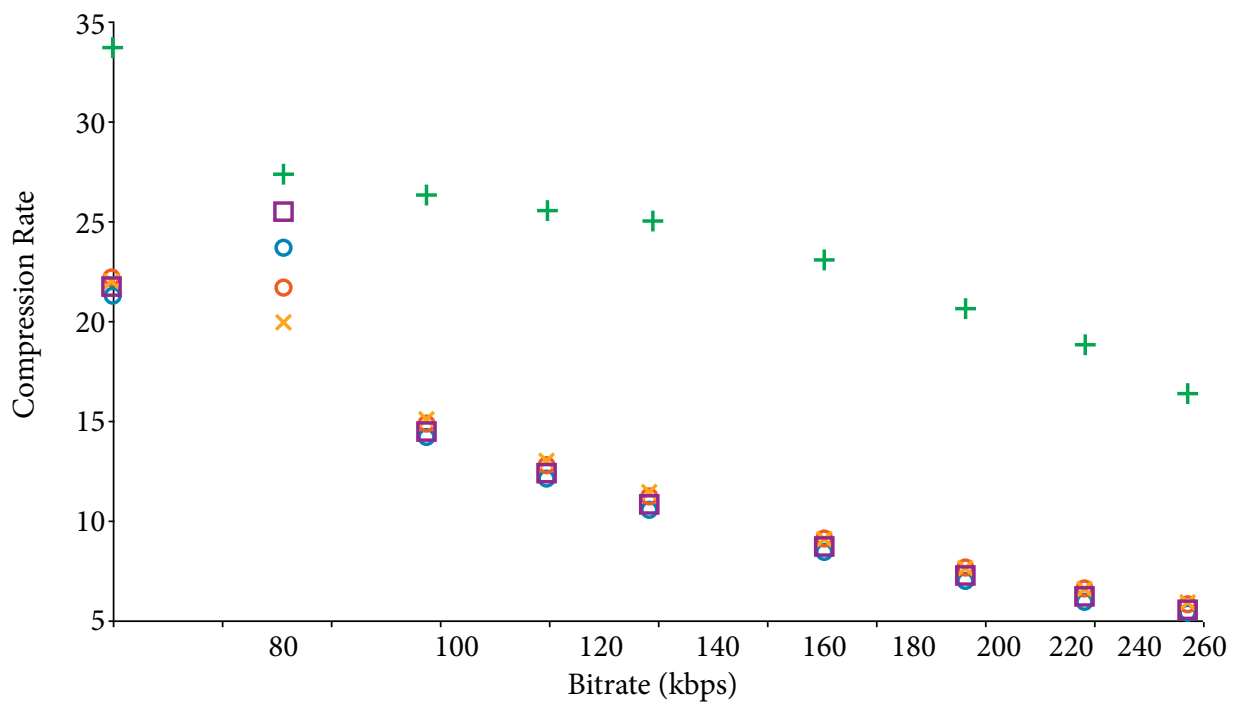

Figure 2. Compression rate for each bitrate.

The Vorbis results up to $1600 \mathrm{~Hz}$ had a linear behavior, where above this value, an increasing trend is noticed, making this format the second-worst for this metric.

Figure 3 show the standard deviation obtained on each one-third octave frequency band using CBR configuration.

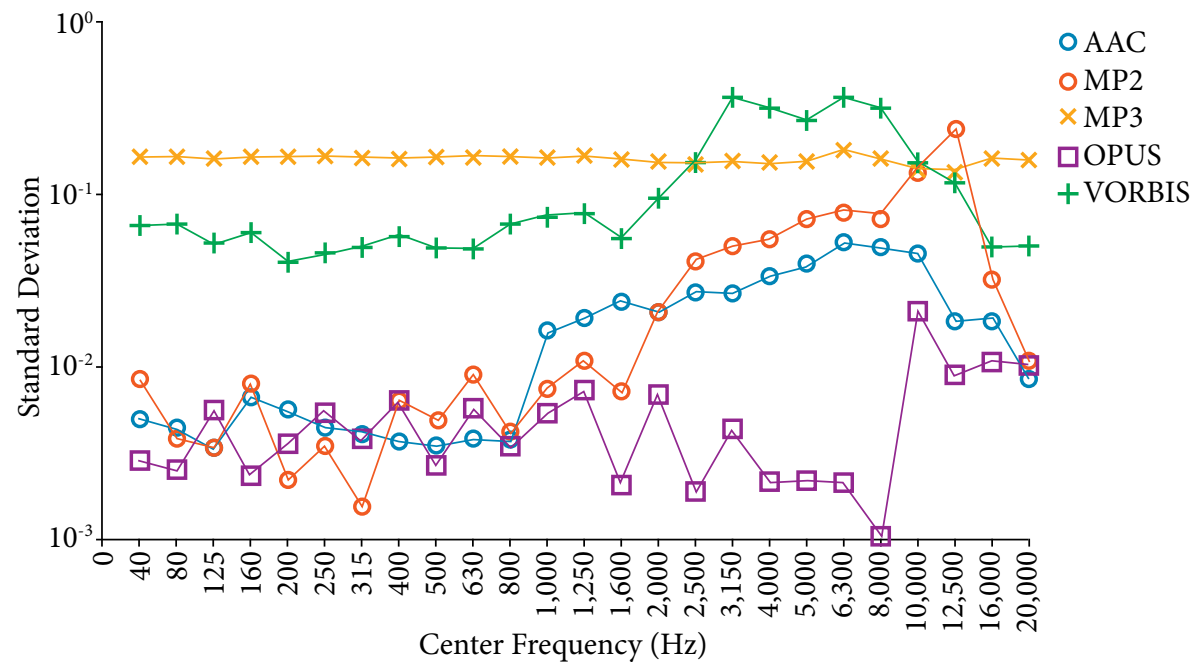

Figure 3. CBR standard deviation results. 


\section{VARIANT BITRATE (VBR) ANALYSIS}

The analysis by using VBR obtained a different bitrate range in comparison to the CBR. The reason for this discrepancy is due to the nature of the variable bitrate, where the quality of the audio file is tuned to use more or fewer bits, thus degrading the audio signal.

Constant bitrate is based on a fixed number of bits used for each second, and the VBR, this value is computed by the quality chosen. The chosen quality has a direct effect on the bitrate used by the compressing code; thus, low quality means lower bitrate.

The MP2 audio format was not considered in the VBR analysis since the resulted bitrate had not changed, thus repeating the same results obtained by using CBR configuration.

Table 1 describes the adopted bit range from each audio format.

Table 1. VBR bit range per audio format.

Audio Format

AAC

MP3

Opus

Vorbis

\section{Bitrate Range [kbps]}

$14-175$

$56-121$

$5-209$

$32-113$

\section{Mean square error (MSE) comparison}

The Opus format has achieved the best results across the entire bitrate, reaching values lower than $1.45 \times 10^{-5}$ for the highest bitrate, and $604.2 \times 10^{-5}$ in the lowest bitrate. The AAC format obtained a value 1500 times higher than AAC at the higher bitrate. The MP2 and MP3 were not considered in this analysis because the number of samples was higher than the original file. Figure 4 shows the mean square error for each lossy audio format.

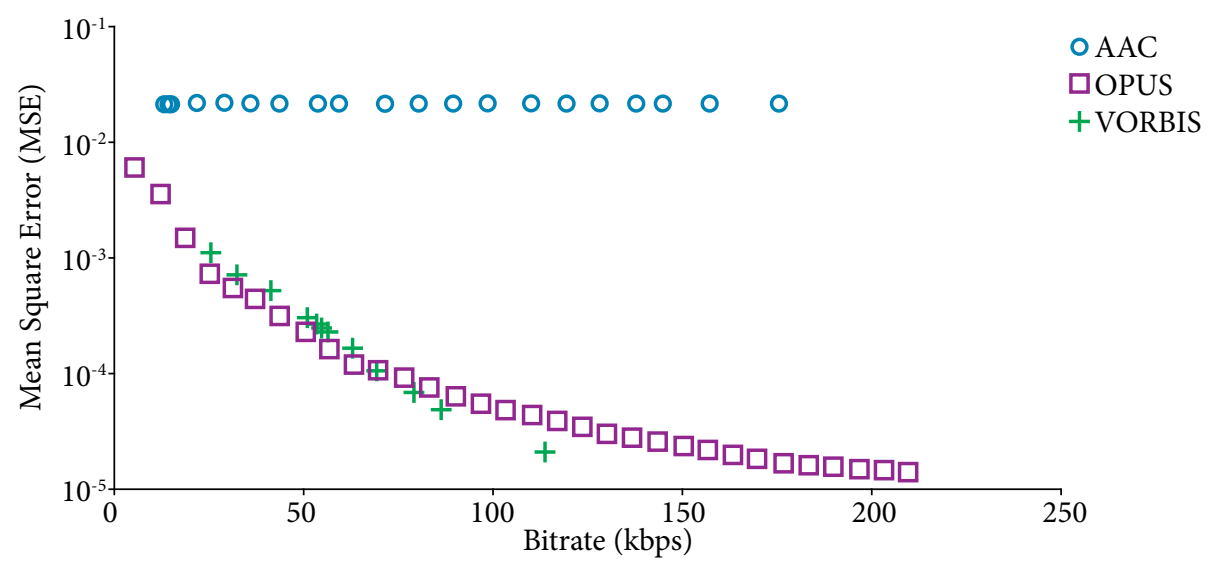

Figure 4. Mean square error for each audio format.

\section{Compression rate (CR) comparison}

Figure 5 displays the compression rate for each audio format by bitrate. The Opus audio format has achieved the best compression across the entire bitrate range, reaching 254:1, saving up to $99.6 \%$ of storage data. The AAC obtained the second-best results at $14 \mathrm{kbps}$, where it has reached 101:1 compression rate. 


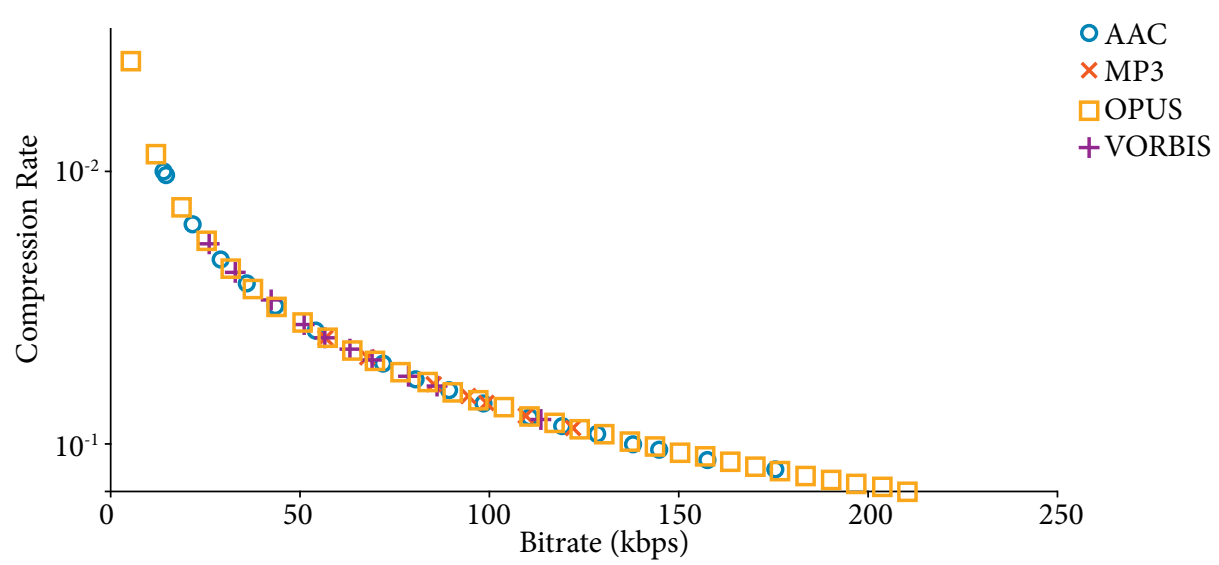

Figure 5. Compression rate for each bitrate.

\section{Sound pressure level}

The computed standard deviation from the SPL results shows that the MP3 had the best results across the entire frequency range. On the other hand, Opus had the worst results up to $5000 \mathrm{~Hz}$, where the AAC presented a slightly higher value from 5000 to $8000 \mathrm{~Hz}$.

The AAC and Vorbis were second and third best results, respectively below $1000 \mathrm{~Hz}$. Above this value, AAC had an inferior response in comparison to Vorbis, except at $3150 \mathrm{~Hz}$ where AAC had a better result than Vorbis. Figure 6 show the standard deviation obtained on each one-third octave frequency band using VBR configuration.

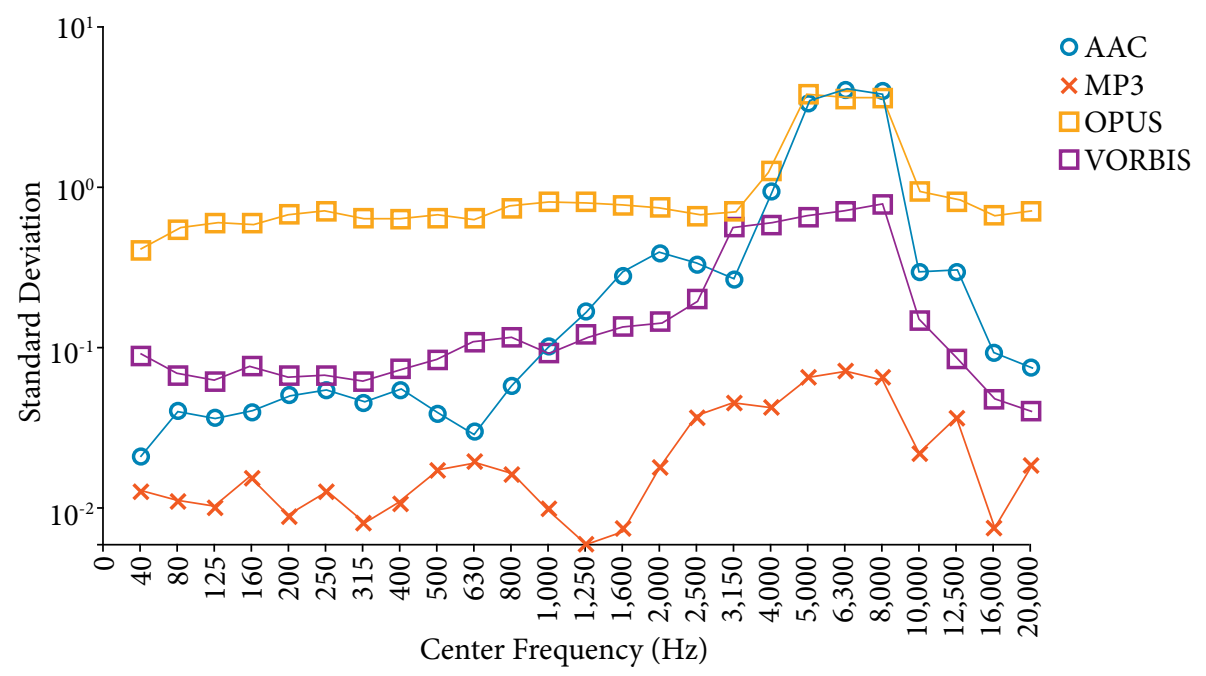

Figure 6. VBR standard deviation results.

\section{CONCLUSION}

The CBR configuration results, the Opus format has achieved the best MSE results across the entire bitrate, reaching values lower than $1.44 \times 10^{-5}$ for the highest bitrate, and $19.46 \times 10^{-5}$ in the lowest bitrate, followed by Vorbis achieving $5.22 \times 10^{-5}$ and $51.02 \times 10^{-5}$ for the higher and lower bitrates, respectively. In the compression rate metric, the Vorbis audio format has achieved the best compression across the entire bitrate range, reaching 33:1, saving up to $97 \%$ storage data. On the other hand, the AAC format presented the lowest compression rate for up to $132 \mathrm{kbps}$, however above this bitrate all the other formats achieved a 
similar result. The computed standard deviation from the SPL results shows that for frequencies below $800 \mathrm{~Hz}$, the Opus, MP2, and AAC presents the best results. Above $800 \mathrm{~Hz}$, only Opus has achieved the lowest results. The MP3 results were the worst up to $2500 \mathrm{~Hz}$, where above this frequency Vorbis presented an increase in the standard deviation. The results above $8000 \mathrm{~Hz}$ are not considered in this analysis, since it is outside the interest range of the study.

The VBR configuration results, the Opus format has achieved the best results across the entire bitrate in the MSE metric, reaching values lower than $1.45 \times 10^{-5}$ for the highest bitrate, and $604.2 \times 10^{-5}$ in the lowest bitrate. The AAC format obtained a value 1500 times higher than $\mathrm{AAC}$ at the higher bitrate. The compression rate results the Opus audio format has achieved the best compression across the entire bitrate range reaching 254:1, saving up to $99.6 \%$ of storage data, followed by AAC at $14 \mathrm{kbps}$ where it has reached 101:1 compression rate. The computed standard deviation from the SPL results shows that the MP3 had the best results across the entire frequency range. The Opus had the worst results up to $5000 \mathrm{~Hz}$, where the AAC presented a slightly higher value from 5000 to $8000 \mathrm{~Hz}$. The AAC and Vorbis were second and third best results, respectively below $1000 \mathrm{~Hz}$. Above this value, AAC had an inferior response in comparison to Vorbis, except at $3150 \mathrm{~Hz}$ where AAC had a better result than Vorbis.

The Vorbis format is the authors' choice using VBR configuration, where it had the best results for the time-domain metric, and a 55:1 compression rate was obtained at $26 \mathrm{kbps}$ bitrate. In the frequency-domain metric, even though this format had the third-best results overall, the values obtained were lower than $1 \%$ of the original value.

\section{AUTHOR'S CONTRIBUTION}

Conceptualization, Corrêa G. C. S. S., Pirk R. and Pinho M. S.; Methodology, Corrêa G. C. S. S., Pirk R. and Pinho M. S.; Investigation, Corrêa G. C. S. S., Pirk R. and Pinho M. S.; Writing - Original Draft, Corrêa G. C. S. S., Pirk R. and Pinho M. S.; Writing - Review and Editing, Corrêa G. C. S. S., Pirk R. and Pinho M. S.; Resources, M Corrêa G. C. S. S., Pirk R. and Pinho M. S.; Supervision, Corrêa G. C. S. S., Pirk R. and Pinho M. S.

\section{ACKNOWLEDGMENTS}

Editors and authors are thankful to Fundação Conrado Wessel for providing the financial support for publishing this article

\section{REFERENCES}

[NASA] National Aeronautics and Space Administration (1986). Report of the PRESIDENTIAL COMMISSION on the Space Shuttle Challenger Accident. Washington: Diane Publishing Co.

Abraham JG, Mishra R, Deepa J (2016) A lossless compression algorithm for vibration data of space systems. Paper presented 2016 International Conference on Next Generation Intelligent Systems (ICNGIS). IEEE; Kottayam, India. https://doi.org/10.1109/ ICNGIS.2016.7854032.

Arenas JP, Margasahayam RN (2006) Noise and vibration of spacecraft structures. Ingeniare Rev Chil Ing 14(3):251-264. https://doi. org/10.4067/S0718-33052006000200009

Barbato L (2008) RTP payload format for Vorbis encoded audio. RFC 5215. https://doi.org/10.17487/rfc5215

Bosi M, Goldberg RE (2003) Introduction to digital audio coding and standards. Boston: Springer. https://doi.org/10.1007/978-1-4615-0327-9

Clark, S (2016) SpaceX lands rocket on ocean-going drone ship [accessed Apr 10 2016]. Available at: http://spaceflightnow. com/2016/04/08/spacex-lands-rocket-on-floating-platform-after-station-resupply-launch/

Dehery YF, Lever M, Urcun P [1991] A MUSICAM source codec for digital audio broadcasting and storage. Paper presented [Proceedings] ICASSP 91: 1991 International Conference on Acoustics, Speech, and Signal Processing. IEEE; Toronto, Canada. https://doi. org/10.1109/ICASSP.1991.151054 
Fastl H, Zwicker E (2007) Psychoacoustics: facts and models. Berlin: Springer. https://doi.org/10.1007/978-3-540-68888-4

Hill P (2018) Audio and speech processing with MATLAB. Boca Raton: CRC Press. https://doi.org/10.1201/9780429444067

ISO (1993a) ISO/IEC 11172-1:1993 Information technology - Coding of moving pictures and associated audio for digital storage media at up to about 1,5 Mbit/s — Part 1: Systems. Geneva: ISO. [accessed Jun 15 2018]. https://www.iso.org/standard/19180.html

ISO (1993b) ISO/IEC 11172-3:1993 Information technology - Coding of moving pictures and associated audio for digital storage media at up to about 1,5 Mbit/s - Part 3: Audio. Geneva: ISO. [accessed Jun 15 2018]. https://www.iso.org/standard/22412.html

ISO (1998) ISO/IEC 13818-3:1998 Information technology — Generic coding of moving pictures and associated audio information — Part 3: Audio. Geneva: ISO. [accessed Jun 15 2018]. https://www.iso.org/standard/26797.html

ISO (2004) ISO/IEC 13818-7:2004 Information technology — Generic coding of moving pictures and associated audio information — Part 7: Advanced Audio Coding (AAC). Geneva: ISO. [accessed Jun 15 2018]. https://www.iso.org/standard/40886.html

Madisetti V (2010) Video, speech, and audio signal processing and associated standards. Boca Raton: CRC Press. https://doi. org/10.1201/9781420046090

McLoughlin IV (2016) Speech and audio processing: A MATLAB ${ }^{\circledR}$-based approach. Cambridge: Cambridge University Press. https://doi. org/10.1017/СВ09781316084205

Meß J-G, Schmidt R, Fey G (2017) Adaptive compression schemes for housekeeping data. Paper presented 2017 IEEE Aerospace Conference. IEEE; Big Sky, United States. https://doi.org/10.1109/AER0.2017.7943580

Nagendra BR, Misra NK, Khan AM (2013) An efficient compression algorithm and a novel file format for satellite vibration test data. Paper presented 2013 IEEE International Conference on Signal Processing, Computing and Control (ISPCC). IEEE; Solan, India. https:// doi.org/10.1109/ISPCC.2013.6663387

Pirk R, Souto CA (2015) Deterministic, hybrid and statistical vibro-acoustic models - a methodology to determine the VLS payload fairing acoustic behavior. J Aerosp Technol Manag 7(1):101-109. https://doi.org/10.5028/jatm.v7i1.392

Selasky HPS (2006) Evaluation of perceptual sound compression with regard to perceived quality and compression methods (Thesis). Grimstad: Agder University College. In English.

Valin JM, Vos K, Terriberry T (2012) Definition of the Opus audio codec. Fremont: Internet Engineering Task Force (IETF). [accessed Nov 25 2016]. https://www.rfc-editor.org/rfc/rfc6716.html

Watkinson J (2004) The MPEG Handbook. 2nd ed. Waltham: Focal Press.

Xiph.Org Foundation (2015) Vorbis I specification. [accessed Jun 15 2019]. https://xiph.org/vorbis/doc/Vorbis_I_spec.pdf

Xiph.Org Foundation (2016) [accessed Jun 15 2016]. https://www.xiph.org/about/ 